\title{
Pancreatic metastasis from invasive pleomorphic lobular carcinoma of the breast: a rare case report
}

\author{
Xiangjie Sun ${ }^{1,2+}$, Ke Zuo $^{1,2+}$, Dan Huang ${ }^{1,2}$, Baohua Yu ${ }^{1,2}$, Yufan Cheng ${ }^{1,2}$ and Wentao Yang ${ }^{1,2^{*}}$ (D)
}

\begin{abstract}
Background: Invasive pleomorphic lobular carcinoma (PLC) is an aggressive subtype of invasive lobular carcinoma of the breast, which has its own histopathological and biological features. The metastatic patterns for PLC are distinct from those of invasive ductal carcinoma. In addition, pancreatic metastasis from PLC is extremely rare.

Case presentation: We report a rare case of a 48-year-old woman presenting with clinical gastrointestinal symptoms and pancreatic metastasis of PLC. The pancreatic tumor was composed of pleomorphic tumor cells arranged in the form of solid sheets and nests and as single files, with frequent mitotic figures, nucleolar prominence, high nuclear to cytoplasmic ratio and loss of cohesion. The malignant cells were positive for p120 (cytoplasmic) and GATA3 and negative for estrogen receptor, progesterone receptor, human epidermal growth factor receptor 2, E-cadherin, gross cystic disease fluid protein 15 and mammaglobin, which indicated a lobular carcinoma phenotype of the breast. Conclusions: To the best of our knowledge, this is one of the few reported cases in the literature of pancreatic metastasis of invasive lobular carcinoma of the breast, of which the definitive diagnosis was obtained only after surgery. Rare metastasis sites should be considered, particularly, when a patient has a medical history of PLC.
\end{abstract}

Keywords: Pleomorphic lobular carcinoma, Triple-negative breast cancer, GATA3, Metastasis

\section{Background}

Invasive lobular carcinoma (ILC) of the breast is the second most common type of invasive breast carcinoma, representing $8-14 \%$ of all breast carcinomas [1]. ILC can be divided into classic, alveolar, solid, tubulolobular, pleomorphic and mixed subtypes according to histological features [2].It is commonly accepted that invasive pleomorphic lobular carcinoma (PLC) is associated with aggressive tumor behavior and poor clinical outcomes. The metastatic patterns of ILC are distinct from those of invasive ductal carcinoma (IDC), and metastasis to uncommon sites may occur including gastrointestinal or peritoneal metastasis [3].

\footnotetext{
*Correspondence: yangwt2000@163.com

${ }^{\dagger}$ Equal contributors

'Department of Pathology, Fudan University Shanghai Cancer Center, No.2 building, 270 Dong'an Road, Shanghai 200032, China

${ }^{2}$ Department of Oncology, Shanghai Medical College, Fudan University, Shanghai 200032, China
}

(1) Biomed Central

(c) The Author(s). 2017 Open Access This article is distributed under the terms of the Creative Commons Attribution 4.0 International License (http://creativecommons.org/licenses/by/4.0/), which permits unrestricted use, distribution, and reproduction in any medium, provided you give appropriate credit to the original author(s) and the source, provide a link to the Creative Commons license, and indicate if changes were made. The Creative Commons Public Domain Dedication waiver (http://creativecommons.org/publicdomain/zero/1.0/) applies to the data made available in this article, unless otherwise stated.

\section{Case presentation}

This case study was approved by the Institutional Review Board of the ethical committee of Fudan University Shanghai Cancer Center.

A 48-year-old woman presented with epigastric discomfort for several weeks, and the levels of serum tumor markers were normal. Computed tomography (CT) of the abdomen showed a mass (diameter $21.7 \mathrm{~mm}$ ) confined to the neck of the pancreas with low density and two enlarged lymph nodes approximately $8 \mathrm{~mm}$ in size around the pancreas (Fig. 1a). Subsequently, she underwent positron emission tomography/computed tomography (PET/CT). A lytic bony lesion in the caput femoris was found besides the pancreatic mass (Fig. 1b), while there were no signs of metastasis in the axillary and supraclavicular lymph nodes, liver or lung. After consideration of imaging manifestations of malignancy, the patient underwent distal pancreatosplenectomy.

Microscopic analysis revealed that the tumor was composed of pleomorphic cells infiltrating the lobules of the pancreas (Fig. 2a). The malignant cells were 


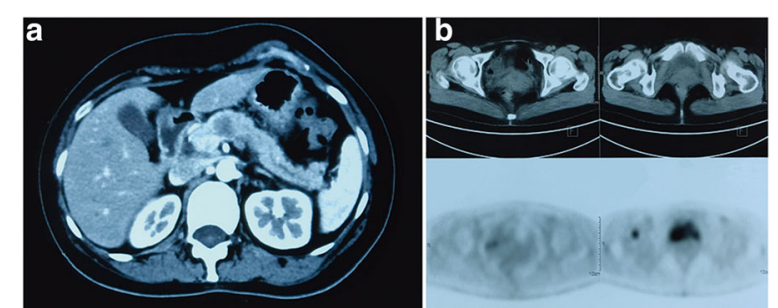

Fig. 1 a: Contrast-enhanced computed tomography scan of the abdomen showing a low-density mass confined to the neck of pancreas. b: Positron emission tomography/computed tomography scan of the pelvis: a lytic bony lesion in the right caput femoris, with abnormal uptake of fluorodeoxyglucose

arranged in the form of solid sheets and nests and as single files, with high nuclear to cytoplasmic ratio and loss of cohesion (Fig. 2b, c). The cell contours were round to polygonal. The cytoplasm of the cells was abundant and eosinophilic, with nuclei of increased size, frequent mitotic figures, and nucleolar prominence
(Fig. 2d). There were no metastases in any of the ten lymph nodes removed around the pancreas.

Immunohistochemical staining revealed that the tumor cells were positive for p120 (cytoplasmic) and GATA3 (Fig. 2e, f) and negative for estrogen receptor (ER), progesterone receptor (PR), human epidermal growth factor receptor 2 (HER2), E-cadherin, gross cystic disease fluid protein 15 (GCDFP-15) and mammaglobin.

Past medical history of this patient was significant for a modified radical mastectomy. Two years ago, a lump was found in her right breast without swelling or pain, and she chose to follow up. The patient presented to our outpatient clinic because of dragging pain in her right breast 10 months later. Physical examination revealed a breast mass (approximately $4 \times 4 \mathrm{~cm}$ ) in the internal inferior quadrant, and invasive carcinoma was confirmed by core needle biopsy. Ultrasonography showed no other metastases in the left breast, supraclavicular lymph nodes or abdomen. The patient was diagnosed with
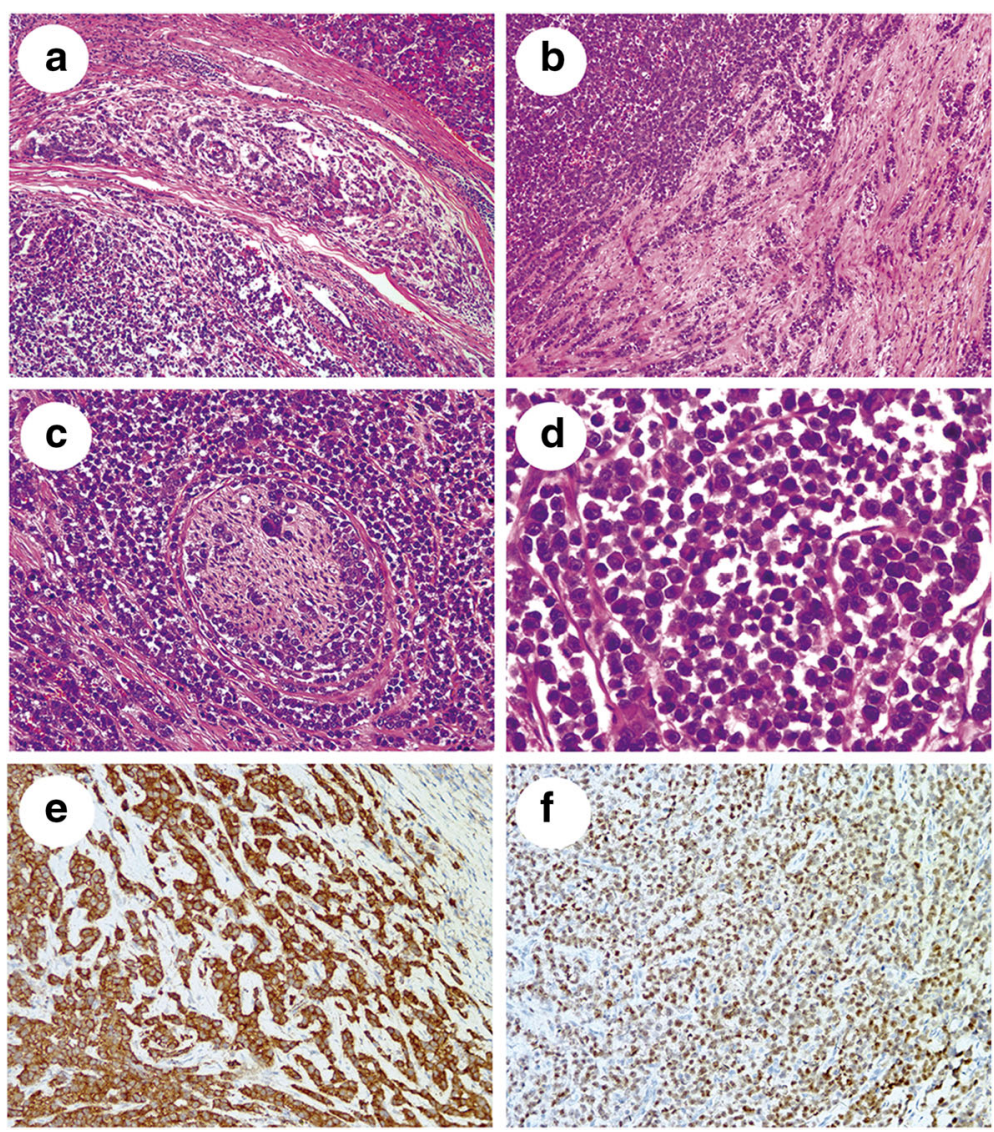

Fig. 2 Photomicrograph of the lesion in pancreas. a: The malignant cells infiltrating the lobules of pancreas $(H \& E, \times 100)$. $\mathbf{b}$ : The tumor cells arranging in the form of solid sheets and single files $(H \& E, \times 100)$. $\mathbf{c}$ : The tumor cells demonstrating a targetoid growth pattern and nerve invasion (H\&E, $\times 200)$. $\mathbf{d}$ High magnification showing the polygonal cell contours, abundant eosinophilic cytoplasm, high nuclear to cytoplasmic ratio and loss of cohesion (H\&E, ×400). e: Diffuse strong cytoplasmic staining for p120 (Envision, ×200). f: Diffuse strong nuclear staining for GATA3 (Envision, ×200) 
invasive PLC $(4.5 \times 3.5 \times 2.0 \mathrm{~cm}$ in size $)$ after modified radical mastectomy (Fig. 3a, b), with the absence of metastases in all 17 lymph nodes removed (pT2 N0 M0, stage IIA). By immunohistochemistry, the tumor showed a triple-negative breast cancer (TNBC) phenotype (ER -/PR-/HER2-), negative for E-cadherin (Fig. 3c), positive for GATA-3 (Fig. 3d), and the Ki-67 index was approximately $40 \%$. After mastectomy, she received adjuvant chemotherapy with paclitaxel for 6 courses and then underwent regular follow up.

The profile of this case is in accordance with the diagnosis of metastatic pleomorphic lobular breast cancer in the pancreas. The patient was then treated with capecitabine, an orally administered chemotherapeutic agent used in the treatment of numerous cancers, including metastatic breast cancer.

\section{Discussion}

As a variant of ILC, invasive PLC is rare, accounting for less than $1 \%$ of all epithelial malignancies of the breast, and associated with older age and postmenopausal status [4-6]. It remains to be determined whether the pleomorphic histology of PLC independently predicts a worse prognosis. Compared to classic ILC, invasive PLC is known to be associated with larger tumors, more frequent lymphovascular invasion, increased lymph node involvement, increased incidence of TNBC, and increased likelihood of distant metastases, which are all unfavorable prognostic factors in breast cancer $[7,8]$.
Unlike most classical ILC demonstrating luminal subtype, ER and PR expression can be both lost in PLC, with PR loss observed in one-third of cases in a majority of studies, and HER2 amplification being found in approximately one-quarter of PLC cases [8]. Compared to IDC, loss of membranous expression for E-cadherin is a defining feature in most ILC cases. A few studies have indicated that loss of E-cadherin expression can be found in $80 \%$ to $100 \%$ of PLC cases [9]. When Ecadherin is dysfunctional, p120, as a member of the Ecadherin complex, accumulates in the cytoplasm, which in turn sets into motion a chemical cascade to increase cell motility [10]. The utilization of both E-cadherin and p120 as markers has been considered the best diagnostic strategy in distinguishing lobular from ductal carcinomas of the breast.

The metastatic pattern for PLC is distinct, the most common sites of which are bone and liver, followed by lung, peritoneum, skin, chest wall and central nervous system $[7,11]$. Pancreatic metastasis from other cancer is rare. Renal cell carcinoma is the most common tumor metastasizing to pancreas, while breast cancer metastasis to pancreas is extremely rare, which forms only a minority of an already small patient population. The majority of the literature devoted to the diagnosis and treatment of these patients consists of anecdotal case reports [12-14]. Previously published series have demonstrated that metastatic involvement of the pancreas from primary breast cancer as solitary metastasis sites has an incidence lower

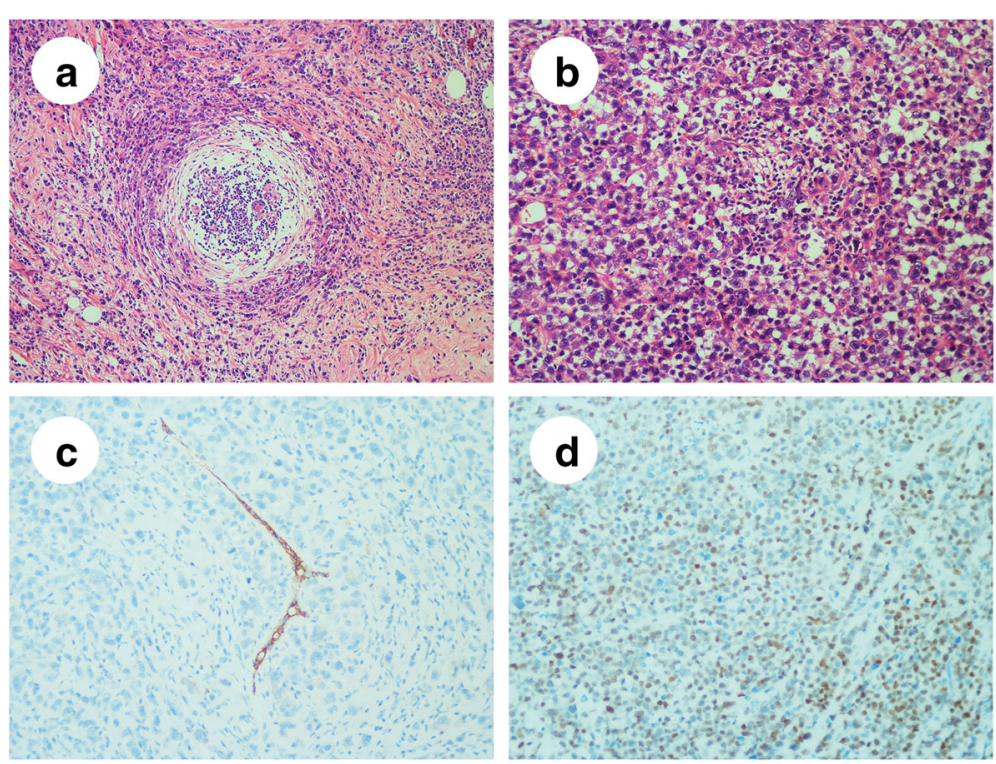

Fig. 3 Photomicrograph of the primary breast lesion. a: The malignant tumor cells showing a targetoid growth pattern or arranging in the form of solid sheets and single files (H\&E, $\times 100)$. b: Abundant and eosinophilic cytoplasm of the cells, with nuclei of increased size and nucleolar prominence $(H \& E, \times 200)$. c: Negative E-cadherin immunostaining of tumor cells with positive internal control in the residual duct (Envision, $\times 200)$. d: Moderate to strong nuclear staining for GATA3 (Envision, $\times 200$ ) 
than 3\% [14]. And almost all the cases that described the pancreatic metastasis were ILC, fewer cases were IDC, none of these was presented with PLC [14-16].Therefore, our report may have the particular meaning in consideration of the differential diagnosis of pancreatic metastasis in case that the prior history is unknown.

It has been reported that ILC metastasizes to organs such as the pancreas, uterus, gastrointestinal tract, urinary tract, reproductive organs and the retroperitoneum after a long disease-free interval [17].In contrast, our patient with PLC had a fairly rapid recurrence, highlighting the potential significant difference in the underlying biology of these two tumors.

Additionally, ILC is seen more often than IDC in cases of breast cancer that is metastatic to the abdominal organs, such as pancreas and gastrointestinal tract [14].The reasons for the different metastatic patterns between ILC and IDC are poorly understood. The loss of expression of E-cadherin in ILC is one possible explanation [18]. It has been demonstrated that loss of expression of E-cadherin in ILC may decrease adhesiveness of cells and facilitate the infiltration through connective tissues and over peritoneal surfaces $[19,20]$. Besides that, in some certain areas with microanatomy, it is more conducive to stop or trap some types of cells with special size or shape. Moreover, the microenvironment of the ovary or peritoneum may provide growth and survival factors that favor ILC cells over IDC cells [19]. The list of anatomical sites associated with ILC metastasis, e.g. ovaries, abdominal cavity, skin and bone, seems like a catalog of tissue compartments with relatively more steroid hormone supply. As compared with the body circulation, estrogen concentrations are much more higher in ovarian tissue and peritoneal cavity fluid [21]. Despite all this,the mechanism involved in ILC metastasis still needs further exploration.

It is difficult to diagnosis metastasis when it occurs in a rare site. Histological characterization is crucial, since the features of PLC are extraordinary. Similar to classic ILC, PLC usually consists of diffuse infiltration of discohesive tumor cells arranged in single files or sheets, and it frequently demonstrates a targetoid growth pattern around terminal ducts and lobules. The nucleus of PLC cells can be nearly four times the size of that of a lymphocyte, while the nucleus of classic ILC cells tends to be only 1-2 times the size of a lymphocyte nucleus [11, 22].

Immunohistochemistry is a reliable method to distinguish between a primary and metastatic tumor. Currently, GATA3 is the most sensitive marker of mammary differentiation, particularly in the setting of metastatic TNBCs, which are typically negative for other traditional mammary-specific markers such as mammaglobin and GCDFP-15. GATA3 positivity has been reported in 73-96\% cases of metastatic breast carcinoma (MBC) [23]. The frequency of GATA3, mammaglobin, and GCDFP-15 was $95 \%, 78 \%$, and $65 \%$, respectively, in a study consisting of $\mathrm{MBC}$ cases $(n=166)$ [24]. GATA3 may have the greatest diagnostic potential in TNBCs. As reported, GATA3 was observed in $44 \%$ to $66 \%$, mammaglobin in $26 \%$, and GCDFP-15 in only $16 \%$ of primary TNBC cases by immunohistochemistry $(n=111)$ [25]. In this case, the primary tumor in the right breast was triplenegative, and the metastatic lesion in pancreas was negative for ER, PR, HER2, mammaglobin and GCDFP-15 but showed diffuse strong staining for GATA3.

Nevertheless, we should pay more attention to the specificity of GATA3 when it is used to distinguish primary pancreatic tumors and metastasis from breast. According to the research of Miettinen et al., besides the strong nuclear GATA3 positivity in primary and metastatic carcinomas of the breast, GATA3 was also expressed in a wide range of tissues and tumors, including pancreatic ductal carcinoma, but in a relative lower frequency (37\%) [26]. Therefore, in differentiating a pancreatic ductal carcinoma from metastatic breast cancer, it is important to use a panel of antibodies instead of one or two antibodies. However, in this case, the diagnosis is straightforward because of the clinical history and special morphology of the tumor cells.

For a pancreatic mass with tumor cells arranged in the form of solid sheets or nests, pancreatic acinar cell carcinoma, pancreatic neuroendocrine tumor, pancreatoblastoma and solid-pseudopapillary neoplasm of the pancreas should also be considered in differential diagnoses. Clinical features, imaging examinations and immunohistochemical tests assist in accurate diagnosis. Additionally, neuroendocrine tumors in pancreas, including low-grade tumors, such as carcinoids and welldifferentiated pancreatic neuroendocrine tumors were negative for GATA3 [26].

Considering the specific management of PLC, there are no guidelines yet. Aggressive managements are expected due to the adverse histopathological and biological features and poorer outcomes. It is accepted that patients with PLC are more likely to require mastectomy than patients with classic ILC, and currently there is a tendency to offer adjuvant chemotherapy for PLC $[5,7,8]$. For metastatic lesions, surgical intervention does not significantly extend overall survival but may be considered as a palliative treatment $[3,27]$.

\section{Conclusion}

The understanding of PLC has progressed dramatically in the past decade. Distal metastasis should be taken into account when a patient has a medical history of invasive PLC, even when a rare metastasis site is involved. 
Histopathological characteristics and immunohistochemical tests are helpful for diagnosis.

\section{Abbreviations}

CT: computed tomography; ER: estrogen receptor; GCDFP-15: gross cystic disease fluid protein 15; HER2: human epidermal growth factor receptor 2; IDC: invasive ductal carcinoma; ILC: invasive lobular carcinoma; PET/ $\mathrm{CT}$ : positron emission tomography/computed tomography (PET/CT); PLC: pleomorphic lobular carcinoma; PR: progesterone receptor; TNBC: triple-negative breast cancer

\section{Acknowledgements}

Not applicable.

\section{Funding}

This research did not receive any specific grant from funding agencies in the public, commercial, or not-for-profit sectors.

\section{Availability of data and materials}

Not applicable.

\section{Authors' contributions}

XJS and KZ conducted data analysis and helped to draft the manuscript. DH, $\mathrm{BHY}$ and YFC collected clinical data and carried out the immunohistochemical staining. WTY revised the manuscript and supervised throughout the study. All authors read and approved the final manuscript.

\section{Ethics approval and consent to participate}

This case study was approved by the Institutional Review Board for ethical committee of Fudan University Shanghai Cancer Center.

\section{Consent for publication}

Written informed consent was obtained from the patient for publication of this case report and any accompanying images. A copy of the written consent is available for review by the Editor-in-Chief of this journal.

\section{Competing interests}

The authors declare no conflicts of interest.

\section{Publisher's Note}

Springer Nature remains neutral with regard to jurisdictional claims in published maps and institutional affiliations.

Received: 15 May 2017 Accepted: 29 June 2017

Published online: 11 July 2017

\section{References}

1. Harake MD, Maxwell AJ, Sukumar SA. Primary and metastatic lobular carcinoma of the breast. Clin Radiol. 2001;56(8):621-30.

2. Sinn HP, Kreipe $H$. A brief overview of the $\mathrm{WHO}$ classification of breast tumors, 4th edition, focusing on issues and updates from the 3rd edition. Breast Care (Basel). 2013;8(2):149-54.

3. McLemore EC, Pockaj BA, Reynolds C, et al. Breast cancer: presentation and intervention in women with gastrointestinal metastasis and carcinomatosis. Ann Surg Oncol. 2005;12(11):886-94.

4. Brogi E, Murray MP, Corben AD. Lobular carcinoma, not only a classic. Breast J. 2010;16(Suppl 1):S10-4.

5. Monhollen L, Morrison C, Ademuyiwa FO, et al. Pleomorphic lobular carcinoma: a distinctive clinical and molecular breast cancer type. Histopathology. 2012;61(3):365-77.

6. Middleton LP, Palacios DM, Bryant BR, et al. Pleomorphic lobular carcinoma: morphology, immunohistochemistry, and molecular analysis. Am J Surg Pathol. 2000;24(12):1650-6.

7. Buchanan CL, Flynn LW, Murray MP, et al. Is pleomorphic lobular carcinoma really a distinct clinical entity? J Surg Oncol. 2008:98(5):314-7.

8. Al-Baimani K, Bazzarelli A, Clemons M, et al. Invasive Pleomorphic lobular carcinoma of the breast: pathologic, clinical, and therapeutic considerations. Clin Breast Cancer. 2015;15(6):421-5.
9. de Deus MR, Wludarski SC, Carvalho FM, et al. Immunohistochemistry applied to the differential diagnosis between ductal and lobular carcinoma of the breast. Appl Immunohistochem Mol Morphol. 2013;21(1):1-12.

10. Dabbs DJ, Bhargava R, Chivukula M. Lobular versus ductal breast neoplasms: the diagnostic utility of p120 catenin. Am J Surg Pathol. 2007;31(3):427-37.

11. Vargas AC, Lakhani SR, Simpson PT. Pleomorphic lobular carcinoma of the breast: molecular pathology and clinical impact. Future Oncol. 2009;5(2):233-43.

12. DeWitt J, Jowell P, Leblanc J, et al. EUS-guided FNA of pancreatic metastases: a multicenter experience. Gastrointest Endosc. 2005;61(6):689-96.

13. Bednar F, Scheiman JM, McKenna BJ, et al. Breast cancer metastases to the pancreas. J Gastrointest Surg. 2013;17(10):1826-31.

14. Molino C, Mocerino C, Braucci A, et al. Pancreatic solitary and synchronous metastasis from breast cancer: a case report and systematic review of controversies in diagnosis and treatment. World J Surg Oncol. 2014;12:2.

15. Mullady DK, Slivka A. Acute pancreatitis secondary to metastatic lobular breast carcinoma: report of a case and review of the literature. Pancreas. 2007;35(2):194-6.

16. Crippa S, Bonardi C, Bovo G, et al. Pancreaticoduodenectomy for pancreatic metastases from breast carcinoma. JOP. 2004;5(5):377-83.

17. Ogino A, Nomizu T, Gonnda K, et al. A case of breast cancer metastasizing to cervix after resection of pancreatic metastasis. Breast Cancer. 2003;10(3): 284-8.

18. Critchley AC, Harvey J, Carr M, et al. Synchronous gastric and colonic metastases of invasive lobular breast carcinoma: case report and review of the literature. Ann R Coll Surg Engl. 2011;93(5):e49-50.

19. Arpino G, Bardou VJ, Clark GM, et al. Infiltrating lobular carcinoma of the breast: tumor characteristics and clinical outcome. Breast Cancer Res. 2004;6(3):R149-56

20. Goldstein NS. Does the level of E-cadherin expression correlate with the primary breast carcinoma infiltration pattern and type of systemic metastases? Am J Clin Pathol. 2002;118(3):425-34.

21. Raap M, Antonopoulos W, Dammrich M, et al. High frequency of lobular breast cancer in distant metastases to the orbit. Cancer Med. 2015;4(1):104-11.

22. Weidner N, Semple JP. Pleomorphic variant of invasive lobular carcinoma of the breast. Hum Pathol. 1992;23(10):1167-71.

23. Asch-Kendrick R, Cimino-Mathews A. The role of GATA3 in breast carcinomas: a review. Hum Pathol. 2016;48:37-47.

24. Sangoi AR, Shrestha B, Yang G, et al. The novel marker GATA3 is significantly more sensitive than traditional markers Mammaglobin and GCDFP15 for identifying breast cancer in surgical and cytology specimens of metastatic and matched primary tumors. Appl Immunohistochem Mol Morphol. 2016;24(4):229-37.

25. Krings G, Nystrom M, Mehdi I, et al. Diagnostic utility and sensitivities of GATA3 antibodies in triple-negative breast cancer. Hum Pathol. 2014; 45(11):2225-32.

26. Miettinen M, McCue PA, Sarlomo-Rikala M, et al. GATA3: a multispecific but potentially useful marker in surgical pathology: a systematic analysis of 2500 epithelial and nonepithelial tumors. Am J Surg Pathol. 2014;38(1):13-22.

27. Zhao R, Li Y, Yu X, et al. Duodenal metastasis from recurrent invasive lobular carcinoma of breast: a case report and literature review. Int J Clin Oncol. 2012:17(2):160-4.

\section{Submit your next manuscript to BioMed Central} and we will help you at every step:

- We accept pre-submission inquiries

- Our selector tool helps you to find the most relevant journal

- We provide round the clock customer support

- Convenient online submission

- Thorough peer review

- Inclusion in PubMed and all major indexing services

- Maximum visibility for your research

Submit your manuscript at www.biomedcentral.com/submit
Biomed Central 